\title{
ADAPTIVE FIXED POINT ITERATIONS FOR SEMILINEAR ELLIPTIC PARTIAL DIFFERENTIAL EQUATIONS
}

\author{
MARIO AMREIN
}

\begin{abstract}
In this paper we study the behavior of finite dimensional fixed point iterations, induced by discretization of a continuous fixed point iteration defined within a Banach space setting. We show that the difference between the discrete sequence and its continuous analogue can be bounded in terms depending on the mesh size of the discretization and the contraction factor, defined by the continuous iteration. Furthermore, we show that the comparison between the finite dimensional and the continuous fixed point iteration naturally paves the way towards a general a posteriori error analysis that can be used within the framework of a fully adaptive solution procedure. In order to demonstrate our approach, we use the Galerkin approximation of singularly perturbed semilinear monotone problems. Our scheme combines the fixed point iteration with an adaptive finite element discretization procedure (based on a robust a posteriori error analysis), thereby leading to a fully adaptive Fixed-Point-Galerkin scheme. Numerical experiments underline the robustness and reliability of the proposed approach.
\end{abstract}

\section{INTRODUCTION}

In this work we study the numerical approximation of problems given by:

$$
\text { find } u \in X: \quad \mathrm{F}(u)=0, \quad \text { in } X^{\prime} \text {, }
$$

where $\mathrm{F}: X \rightarrow X^{\prime}$ signifies a possibly nonlinear operator. Here, $X$ stands for a real Hilbert space, with inner product denoted by $(\cdot, \cdot)_{X}$ and induced norm $\|x\|_{X}=\sqrt{(x, x)_{X}}$. Furthermore, $X^{\prime}$ signifies the dual of $X$.

Fixed Point Galerkin Methods. As a result of the possible nonlinearity of $\mathrm{F}$, for a given $u^{0} \in X$, we consider the fixed point iteration

$$
\left(u^{n+1}, v\right)_{X}=\mathrm{B}\left(u^{n}\right)(v), \quad \forall v \in X,
$$

where, for a fixed $t>0$, the operator $\mathrm{B}$ is defined by

$$
\mathrm{B}(u)(v):=(u, v)_{X}-t \cdot \mathbf{F}(u)(v), \quad \forall v \in X .
$$

To guarantee the convergence of the above iteration, we suppose that $F$ satisfies the following two assumptions, namely the Lipschitz continuity property

$$
|\mathrm{F}(x)(v)-\mathrm{F}(y)(v)| \leq L\|x-y\|_{X}\|v\|_{X}, \quad L>0,
$$

as well as the strong monotonicity property

$$
(\mathrm{F}(x)-\mathrm{F}(y))(x-y) \geq c\|x-y\|_{X}^{2}, \quad c>0 .
$$

Under these assumptions, it is well known that there exists a unique solution $u \in X$ solving (1) (see, e.g. [21], or section 2 in this work). More precisely, it can be shown that the operator defined in (3) is contractive, i.e., there holds

$$
|\mathrm{B}(x)(v)-\mathrm{B}(y)(v)| \leq \alpha\|x-y\|_{X}\|v\|_{X}, \quad \alpha=\sqrt{1-\left(\frac{c}{L}\right)^{2}} \in(0,1),
$$

\footnotetext{
Applied University of Zürich, CH-8004 Switzerland

E-mail address: mario.amrein@hslu.ch.

2010 Mathematics Subject Classification. 62F35, 35J61, 65N30, 65L11.

Key words and phrases. Adaptive fixed point methods, a posteriori error analysis, strongly monotone problems, semilinear elliptic problems, singularly perturbed problems, adaptive finite element methods.
} 
and therefore, based on Banach's fixed point Theorem, the solution can be obtained by iterating (2) with optimal step size $t_{\text {opt }}=\frac{c}{L^{2}}$ (see also [9]).

However in actual computations, we can only solve a finite dimensional analogue of equation (2). More precisely, let $X_{h} \subset X$ be a linear finite dimensional subspace of $X$. We then observe

$$
\left.\mid \mathrm{B}\left(x_{h}\right)\left(v_{h}\right)-\mathrm{B}\left(y_{h}\right)\right)\left(v_{h}\right) \mid \leq \alpha_{h}\left\|x_{h}-y_{h}\right\|_{X}\left\|v_{h}\right\|_{X},
$$

with $0<\alpha_{h} \leq \alpha$ from where we get the existence of a unique $x_{h} \in X_{h}$ such that $\mathrm{B}\left(x_{h}\right)\left(v_{h}\right)=$ $\left(x_{h}, v_{h}\right)_{X} \forall v_{h} \in X_{h}$ and solving

$$
\mathrm{F}\left(x_{h}\right)\left(v_{h}\right)=0, \quad \forall v_{h} \in X_{h} .
$$

For a given initial value $u_{0}^{h} \in X_{h}$, the solution can be obtained by the fixed point iteration

$$
\left(u_{h}^{n+1}, v_{h}\right)_{X}=\mathrm{B}\left(u_{h}^{n}\right)\left(v_{h}\right), \quad \forall v_{h} \in X_{h} .
$$

In order to establish a possible a priori error analysis, we will focus (following the argument from $[10, \S 8.1]$ for Newton's method) on the distance, between the sequence $\left\{u^{n}\right\}_{n \geq 0}$, corresponding to the iteration from (2), and its discrete analogue $\left\{u_{h}^{n}\right\}_{n \geq 0}$ defined in (5). More precisely: we will show that the discrete sequence tracks its continuous analogue with a maximal distance, which can be bounded in terms depending on the mesh size parameter $h>0$ and the contraction factor $\alpha \in(0,1)$ defined in (4). As it turns out, the above outlined approach naturally paves the way towards an a posteriori error analysis, where two different error indicators contribute to the $a$ posteriori error bound. They are caused by the nonlinearity of the problem and its discretization.

Based on these two error contributions, and following along the lines of [9], we formulate an adaptive procedure. More specifically: as long as our adaptive porcedure is running, we either perform a fixed point iteration or refine the current space $X_{h}$ based on the derived error indicators. In order to test such a fully adaptive procedure, we concentrate on semilinear elliptic boundary value problems with possible singular perturbations.

Linearization schemes. Let us briefly address two possible strategies when solving nonlinear problems numerically. Firstly, the nonlinear problem can be formulated within an accurate finite dimensional framework. Based on a suitable iterative scheme, the resulting nonlinear finite dimensional problem will be linearized. Alternatively, a local linearization is applied. This leads to a sequence of linear problems which afterwards will be discretized by some suitable numerical approximation schemes. It is noteworthy that the second approach offers the application of the existing numerical analysis and the computational techniques for linear problems (such as e.g. the development of classical residual-based error bounds). The concept of approximating infinite dimensional nonlinear problems by appropriate linear discretization schemes has been studied by several authors in the recent past. For example, the approach presented in [9] (see also the work $[8,16]$ ) combines fixed point linearization methods and Galerkin approximations in the context of strictly monotone problems. Similarly, in $[1,2,4,12,13,17]$, the nonlinear PDE problems at hand are linearized by an (adaptive) Newton technique, and subsequently discretized by a linear finite element method. On a related note, the discretization of a sequence of linearized problems resulting from the local approximation of semilinear evolutionary problems has been investigated in [3]. In all of the works [1-4,9], the key idea in obtaining fully adaptive discretization schemes is to provide a suitable interplay between the underlying linearization procedure and (adaptive) Galerkin methods; this is based on investing computational time into whichever of these two aspects is currently dominant.

Outline. The outline of this paper is as follows. In Section 2 we study fixed point iterations within the context of general Hilbert spaces and derive an apriori and a posteriori error analysis. Subsequently, the purpose of Section 3 is the discretization of the resulting sequence of linear problems by the finite element method and the development of an $\varepsilon$-robust a posteriori error analysis. The final estimate (Theorem 3.3) bounds the error in terms of the (elementwise) finite element approximation (FEM-error) and the error caused by the fixed point iteration of the original problem. Then, in order to define a fully adaptive Fixed-Point-Galerkin scheme, we propose an interplay between the adaptive method and the adaptive finite element approach: More precisely, 
as the adaptive procedure is running, we either perform a fixed point iteration or refine the current finite element mesh based on the a posteriori error estimate (Section 3); this is carried out depending on which of the errors (FEM-error or fixed point error) is more dominant in the present iteration step. In Section 4 we provide a numerical experiment which shows that the proposed scheme is reliable and $\varepsilon$-robust for reasonable choices of initial guesses. Finally, we summarize and comment our findings in Section 5.

\section{APRIORI AND A POSTERIORI ERROR ESTIMATES}

First of all and with the purpose of completness, we recall the well known Banach's fixed point Theorem (see, e.g. [14]), which asserts that any Lipschitz continuous map $\mathcal{B}: X \rightarrow X$ with Lipschitz constant $\alpha \in(0,1)$, and operating over a Banach space $X$, possesses a unique fixed point. Furthermore, for a given initial guess $x_{0} \in X$, the fixed point can be obtained through iteration of $x_{n+1}=\mathcal{B}\left(x_{n}\right) \quad n \geq 0$. Incidentally, there holds the following apriori error estimate

$$
\left\|x-x_{n}\right\|_{X} \leq \frac{\alpha^{n}}{1-\alpha}\left\|x_{0}-x_{1}\right\|_{X} .
$$

We now show that the difference $u_{h}^{n}-u^{n}$ between the discrete sequence $\left\{u_{h}^{n}\right\}_{n \geq 0}$ and its continuous analogue $\left\{u^{n}\right\}_{n \geq 0}$ is bounded by the discretization error and the contraction constant $\alpha \in(0,1)$. Indeed, there holds the following result:

Theorem 2.1. For given initial values $u_{h}^{0}=u^{0} \in X_{h} \subset X$ we consider the iterations

$$
\left(u^{n+1}, v\right)_{X}=\mathrm{B}\left(u^{n}\right)(v) \quad \forall v \in X, \quad\left(u_{h}^{n+1}, v_{h}\right)_{X}=\mathrm{B}\left(u_{h}^{n}\right)\left(v_{h}\right) \quad \forall v_{h} \in X_{h} .
$$

Assume that the disretization of $X$ is fine enough such that

$$
\left|\mathrm{B}\left(u_{h}^{n}\right)(v)-\left(u_{h}^{n+1}, v\right)_{X}\right| \leq \eta_{h}\|v\|_{X}, \quad \forall v \in X .
$$

Then there holds

$$
\left\|u^{n+1}-u_{h}^{n+1}\right\|_{X} \leq \frac{\eta_{h}}{1-\alpha} \text { for all } n \in \mathbb{N} .
$$

Proof. We proceed along the lines of $[10, \S 8.1]$, where the authors show a similar result for Newton's method.

The argument is inductive. Therefore, given a sequence $\left\{\varepsilon_{k}\right\}_{k \geq 0} \subset \mathbb{R}_{\geq 0}$ and assuming that there holds

$$
\left\|u^{n}-u_{h}^{n}\right\|_{X} \leq \varepsilon_{n} .
$$

Since $u^{0}=u_{h}^{0}$, we can choose $\varepsilon_{0}=0$ for $n=0$. For $n \geq 0$, we further introduce sequences $\left\{\tilde{u}^{k, n}\right\}_{k \geq n}$ generated by the iteration

$$
\left(\tilde{u}^{k+1, n}, v\right)_{X}=\mathrm{B}\left(\tilde{u}^{k, n}\right)(v), \quad \tilde{u}^{n, n}:=u_{h}^{n}, \quad v \in X .
$$

We note that these sequences start at the discrete points $u_{h}^{n}$ and are convergent to the unique zero $u^{\infty}$ of $\mathrm{F}$ (see Figure 1).

Induction step: employing the triangle inequality we get

$$
\left\|u^{n+1}-u_{h}^{n+1}\right\|_{X} \leq\left\|u^{n+1}-\tilde{u}^{n+1, n}\right\|_{X}+\left\|\tilde{u}^{n+1, n}-u_{h}^{n+1}\right\|_{X} .
$$

The first term can be estimated using (9) and the $\alpha$-Lipschitz continuity of B:

i.e., we have

$$
\begin{aligned}
\left\|u^{n+1}-\tilde{u}^{n+1, n}\right\|_{X}^{2} & =\left(u^{n+1}-\tilde{u}^{n+1, n}, u^{n+1}-\tilde{u}^{n+1, n}\right)_{X} \\
& =\left(\mathrm{B}\left(u^{n}\right)-\mathrm{B}\left(\tilde{u}^{n, n}\right)\right)\left(u^{n+1}-\tilde{u}^{n+1, n}\right) \\
& \leq \alpha\left\|u^{n}-\tilde{u}^{n, n}\right\|_{X}\left\|u^{n+1}-\tilde{u}^{n+1, n}\right\|_{X} \\
& =\alpha\left\|u^{n}-u_{h}^{n}\right\|_{X}\left\|u^{n+1}-\tilde{u}^{n+1, n}\right\|_{X} \\
& \leq \alpha \varepsilon_{n}\left\|u^{n+1}-\tilde{u}^{n+1, n}\right\|_{X},
\end{aligned}
$$

$$
\left\|u^{n+1}-\tilde{u}^{n+1, n}\right\|_{X} \leq \alpha \varepsilon_{n}
$$




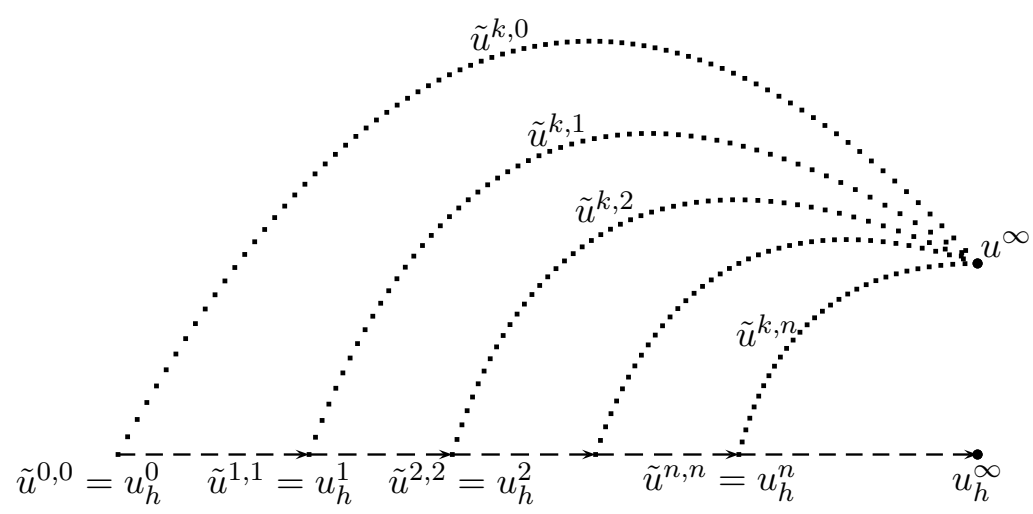

Figure 1. The sequence given in (10).

For the second term $\left\|\tilde{u}^{n+1, n}-u_{h}^{n+1}\right\|_{X}$ in (11) we use (8) and observe:

$$
\begin{aligned}
\left\|\tilde{u}^{n+1, n}-u_{h}^{n+1}\right\|_{X}^{2}= & \left(\tilde{u}^{n+1, n}, \tilde{u}^{n+1}-u_{h}^{n+1}\right)_{X}-\mathrm{B}\left(u_{h}^{n}\right)\left(\tilde{u}^{n+1, n}-u_{h}^{n+1}\right) \\
& +\mathrm{B}\left(u_{h}^{n}\right)\left(\tilde{u}^{n+1, n}-u_{h}^{n+1}\right)-\left(u_{h}^{n+1}, \tilde{u}^{n+1, n}-u_{h}^{n+1}\right)_{X} \\
\leq & \left|\mathrm{B}\left(u_{h}^{n}\right)\left(\tilde{u}^{n+1, n}-u_{h}^{n+1}\right)-\left(u_{h}^{n+1}, \tilde{u}^{n+1, n}-u_{h}^{n+1}\right)_{X}\right| \\
\leq & \eta_{h}\left\|\tilde{u}^{n+1, n}-u_{h}^{n+1}\right\|_{X},
\end{aligned}
$$

and therefore

$$
\left\|\tilde{u}^{n+1, n}-u_{h}^{n+1}\right\|_{X} \leq \eta_{h} .
$$

Taking (12) into account we can bound (11) by

$$
\left\|u^{n+1}-u_{h}^{n+1}\right\|_{X} \leq \alpha \varepsilon_{n}+\eta_{h}=: \varepsilon_{n+1} .
$$

We now consider the fixed point iteration (see Figure 2)

$$
\varepsilon_{n+1}=\alpha \varepsilon_{n}+\eta_{h}, \quad \varepsilon_{0}=0 .
$$

Since $\alpha \in(0,1)$ we conlude that $\varepsilon_{n} \leq \varepsilon_{\infty}=\frac{\eta_{h}}{1-\alpha}$ for all $n \geq 0$ which completes the inductive and therefore the whole argument.

Based on this result, there holds the following apriori bound:

Corollary 2.2. For any initial value $u^{0} \in X_{h}$ there holds the following apriori error estimate:

$$
\left\|u-u_{h}^{n}\right\|_{X} \leq \frac{1}{1-\alpha}\left(\alpha^{n}\left\|u^{1}-u^{0}\right\|_{X}+\eta_{h}\right) .
$$

Proof. By virtue of the triangle inequality and employing (6) together with (8) we obtain

$$
\left\|u-u_{h}^{n}\right\|_{X} \leq\left\|u-u^{n}\right\|_{X}+\left\|u^{n}-u_{h}^{n}\right\|_{X} \leq \frac{1}{1-\alpha}\left(\alpha^{n}\left\|u^{1}-u^{0}\right\|_{X}+\eta_{h}\right) .
$$

Based on the Lipschitz continuity of $F$ we readily infer

$$
\left\|\mathrm{F}\left(u_{h}^{n}\right)\right\|_{X^{\prime}}=\left\|\mathrm{F}(u)-\mathrm{F}\left(u_{h}^{n}\right)\right\|_{X^{\prime}} \leq L\left\|u-u_{h}^{n}\right\|_{X} .
$$

Thus we can bound the residual $\mathrm{F}\left(u_{h}^{n}\right)$ as follows:

Corollary 2.3. There holds

$$
\left\|\mathrm{F}\left(u_{h}^{n}\right)\right\|_{X^{\prime}} \leq \frac{L}{1-\alpha}\left(\alpha^{n}\left\|u^{1}-u^{0}\right\|_{X}+\eta_{h}\right), \quad\|\mathrm{F}(x)\|_{X^{\prime}}:=\sup _{\|v\|_{X}=1} \mathrm{~F}(x)(v) .
$$




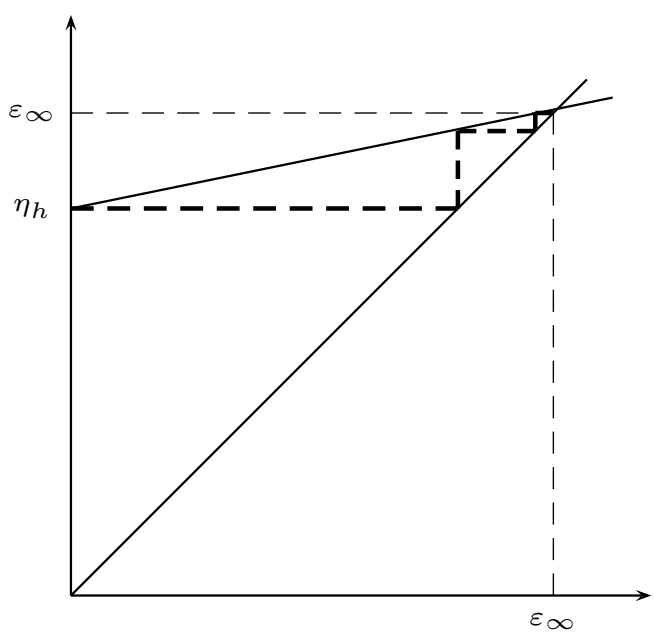

Figure 2. The fixed point iteration given in (15).

2.1. A posteriori error analysis. Following along the lines of [9], we now want to exploit an a posteriori error analysis for strictly monotone operators. In doing so, we first recall the well known Theorem of Zarantonello (see [21]):

Theorem 2.4. Suppose that $\mathrm{F}: X \rightarrow X^{\prime}$ is L-Lipschitz and strongly monotone, with monotonicity constant $c$. Then there exists a unique zero for $\mathrm{F}$.

Proof. Let $\mathrm{J}(x)(y)=(x, y)_{X}$ denote the Riesz-Isometry $\mathrm{J}: X \rightarrow X^{\prime}$. We show that $\mathcal{B}: X \rightarrow X$ given by $\mathcal{B}(x):=\mathrm{J}^{-1}(\mathrm{~B}(x))$ is $\alpha$-contractive and therefore possesses a unique fixed point $u$ which is the desired zero for the operator $\mathcal{F}(x):=\mathrm{J}^{-1}(\mathrm{~F}(x))$.

We have

$$
\|\mathcal{B}(x)-\mathcal{B}(y)\|_{X}^{2}=\|x-y\|_{X}^{2}-2 t(x-y, \mathcal{F}(x)-\mathcal{F}(y))_{X}+t^{2}\|\mathcal{F}(x)-\mathcal{F}(y)\|_{X}^{2} .
$$

Notice that

and

$$
\|\mathcal{F}(x)-\mathcal{F}(y)\|_{X}=\|\mathrm{F}(x)-\mathrm{F}(y)\|_{X^{\prime}} \leq L\|x-y\|_{X},
$$

$$
(\mathrm{F}(x)-\mathrm{F}(y))(x-y)=\mathrm{J}(\mathcal{F}(x)-\mathcal{F}(y))(x-y)=(\mathcal{F}(x)-\mathcal{F}(y), x-y)_{X} \geq c\|x-y\|_{X}^{2} .
$$

Thus we arrive at

$$
\|\mathcal{B}(x)-\mathcal{B}(y)\|_{X}^{2} \leq f(t) \cdot\|x-y\|_{X}^{2}
$$

with $f(t):=1-2 c t+(L t)^{2}$. Moreover we observe that for $t \in\left(0,2 c / L^{2}\right)$ we have $\sqrt{f(t)}<1$. Note that the optimal contraction constant is $\alpha_{\mathrm{opt}}:=f\left(t_{\mathrm{opt}}\right)=\sqrt{1-c^{2} / L^{2}}$ with $t_{\mathrm{opt}}:=\frac{c}{L^{2}}$.

Based on this result there holds the following a posteriori error estimate:

Proposition 2.5. If $\mathrm{F}: X \rightarrow X^{\prime}$ satisfies (A1) and (A2), then for $e_{h}^{n+1}:=u-u_{h}^{n+1}$, there holds the a posteriori error bound:

$$
\left\|e_{h}^{n+1}\right\|_{X} \leq \frac{L^{2}}{c^{2}} \cdot \eta_{h}+\frac{L}{c}\left(1+\frac{L}{c}\right) \cdot\left\|u_{h}^{n+1}-u_{h}^{n}\right\|_{X} .
$$

Proof. We follow along the lines of [9]. Recalling the coercivity of $\mathcal{F}$ we get

$$
c\left\|e_{h}^{n+1}\right\|_{X}^{2} \leq-\left(\mathcal{F}\left(u_{h}^{n+1}\right), e_{h}^{n+1}\right)_{X} .
$$


Next, we recall the sequences $\left\{\tilde{u}^{k, n}\right\}_{k \geq n}$ given in (10), i.e., we observe

$$
\left(\tilde{u}^{n+1, n}, e_{h}^{n+1}\right)_{X}=\left(\mathcal{B}\left(\tilde{u}^{n, n}\right), e_{h}^{n+1}\right)_{X}=\left(\mathcal{B}\left(u_{h}^{n}\right), e_{h}^{n+1}\right)_{X}=\left(u_{h}^{n}, e_{h}^{n+1}\right)_{X}-t\left(\mathcal{F}\left(u_{h}^{n}\right), e_{h}^{n+1}\right)_{X} .
$$

By virtue of the Cauchy-Schwarz inequality and the Lipschitz continuity of $\mathcal{F}$ we get

$$
\begin{aligned}
& c\left\|e_{h}^{n+1}\right\|_{X}^{2} \\
\leq & -\left(\mathcal{F}\left(u_{h}^{n+1}\right), e_{h}^{n+1}\right)_{X}+t^{-1}\left(\tilde{u}^{n+1, n}-u_{h}^{n}, e_{h}^{n+1}\right)_{X}+\left(\mathcal{F}\left(u_{h}^{n}\right), e_{h}^{n+1}\right)_{X} \\
= & t^{-1}\left(\tilde{u}^{n+1, n}-u_{h}^{n+1}, e_{h}^{n+1}\right)_{X}+t^{-1}\left(u_{h}^{n+1}-u_{h}^{n}, e_{h}^{n+1}\right)_{X}+\left(\mathcal{F}\left(u_{h}^{n}\right), e_{h}^{n+1}\right)_{X}-\left(\mathcal{F}\left(u_{h}^{n+1}\right), e_{h}^{n+1}\right)_{X} \\
\leq & t^{-1}\left(\left\|\tilde{u}^{n+1, n}-u_{h}^{n+1}\right\|_{X}+\left\|u_{h}^{n+1}-u_{h}^{n}\right\|_{X}\right)\left\|e_{h}^{n+1}\right\|_{X}+L\left\|u_{h}^{n+1}-u_{h}^{n}\right\|_{X}\left\|e_{h}^{n+1}\right\|_{X} .
\end{aligned}
$$

Dividing by $c\left\|e_{h}^{n+1}\right\|_{X}$ and using $t:=\frac{c}{L^{2}}$ we obtain

$$
\left\|e_{h}^{n+1}\right\|_{X} \leq \frac{L^{2}}{c^{2}}\left\|\tilde{u}^{n+1, n}-u_{h}^{n+1}\right\|_{X}+\frac{L}{c}\left(1+\frac{L}{c}\right)\left\|u_{h}^{n+1}-u_{h}^{n}\right\|_{X} .
$$

Recalling (14) we conclude (17).

\section{Application to SEMilinear PRoblems}

3.0.1. Problem formulation. In this section, we focus on the numerical approximation procedure for semilinear elliptic boundary value problems with possible singular perturbations. More precisely, for a fixed parameter $\varepsilon>0$ (possibly with $\varepsilon \ll 1$ ), and a continuous function $f: \mathbb{R} \rightarrow \mathbb{R}$, we consider the problem of finding $u: \Omega \rightarrow \mathbb{R}$ that satisfies

$$
\begin{aligned}
-\varepsilon \Delta u & =f(x, u), & & \text { in } \Omega, \\
u & =0, & & \text { on } \partial \Omega .
\end{aligned}
$$

Here, $\Omega \subset \mathbb{R}^{d}$, with $d=1$ or $d=2$, is an open and bounded $1 \mathrm{~d}$ interval or a $2 \mathrm{~d}$ Lipschitz polygon, respectively. Problems of this type appear in a wide range of applications including, e.g., nonlinear reaction-diffusion in ecology and chemical models $[7,11,15,18,19]$, economy [5], or classical and quantum physics $[6,20]$.

In this work, we are interested in a unique solution $u \in X:=H_{0}^{1}(\Omega)$ solving (18): here, we denote by $H_{0}^{1}(\Omega)$ the standard Sobolev space of functions in $H^{1}(\Omega)=W^{1,2}(\Omega)$ with zero trace on $\partial \Omega$. Furthermore, the weak formulation of (18) is to find $u \in X:=H_{0}^{1}(\Omega)$ such that

$$
\mathrm{F}_{\varepsilon}(u)(v)=0, \quad \forall v \in X,
$$

where

$$
\mathrm{F}_{\varepsilon}(u)(v):=\int_{\Omega}\{\varepsilon \nabla u \nabla v-f(u) v\} \mathrm{d} \boldsymbol{x} .
$$

In addition, we introduce the inner product

$$
(u, v)_{X}:=\int_{\Omega}\{u v+\varepsilon \nabla u \cdot \nabla v\} \mathrm{d} \boldsymbol{x}, \quad u, v \in X,
$$

with induced norm on $X$ given by

$$
\|u\|_{\varepsilon, D}:=\left(\varepsilon\|\nabla u\|_{0, D}^{2}+\|u\|_{0, D}^{2}\right)^{1 / 2}, \quad u \in H^{1}(D),
$$

where $\|\cdot\|_{0, D}$ denotes the $L^{2}$-norm on $D$. Frequently, for $D=\Omega$, the subindex ' $D$ ' will be omitted. Note that in the case of $f(u)=-u+g$, with $g \in L^{2}(\Omega)$, i.e., when (18) is linear and strongly elliptic, the norm $\|\cdot\|_{\varepsilon, \Omega}$ is a natural energy norm on $X$.

In what follows we shall use the abbreviation $x \preccurlyeq y$ to mean $x \leq c y$, for a constant $c>0$ independent of the mesh size $h$ and of $\varepsilon>0$. 
3.0.2. Fixed-Point-Iteration. For $u^{n} \in X$ and $t>0$, the fixed-point iteration is to find $u^{n+1} \in X$ from $u^{n}$ such that

$$
\left(u^{n+1}, v\right)_{X}=\mathrm{B}_{\varepsilon}\left(u^{n}\right)(v), \quad \forall v \in X,
$$

where for fixed $u \in X, t>0$, we set

$$
\mathrm{B}_{\varepsilon}(u)(v):=(u, v)_{X}-t \int_{\Omega}\{\varepsilon \nabla u \cdot \nabla v-f(u) v\} \mathrm{d} \boldsymbol{x} .
$$

Remark 3.1. We assume that $f$ is Lipschitz continuous with Lipschitz constant $L_{f}$. Furthermore we need the following monotonicity property:

$$
(f(x)-f(y))(x-y) \leq-c_{f}(x-y)^{2}, \quad c_{f}>0 .
$$

As a consequence, the operator $F$ in (19) is Lipschitz continuous and strongly monotone with Lipschitz constant $L=\max \left(1, L_{f}\right)$ and monotonincity constant $c=\min \left(1, c_{f}\right)$ respectively. In particular, applying the iteration given in (20), we obtain a unique fixed point $x \in X$ for $\mathrm{B}_{\varepsilon}$ which is the unique root of $F_{\varepsilon}$ given in (19).

3.1. Galerkin Discretization. In order to provide a numerical approximation of (18), we will discretize the linear weak formulation (20) by means of a finite element method, which constitutes a Fixed-Point-Galerkin approximation scheme. Furthermore, we shall derive a posteriori error estimates for the finite element discretization which allow for an adaptive refinement of the meshes in each iteration step. This, together with the a posteriori error estimate from Proposition 2.5 leads to a fully adaptive Fixed-Point-Galerkin discretization method for (20).

3.1.1. Finite Element Meshes and Spaces. Let $\mathcal{T}^{h}=\{T\}_{T \in \mathcal{T}^{h}}$ be a regular and shape-regular mesh partition of $\Omega$ into disjoint open simplices, i.e., any $T \in \mathcal{T}^{h}$ is an affine image of the (open) reference simplex $\widehat{T}=\left\{\widehat{x} \in \mathbb{R}_{+}^{d}: \sum_{i=1}^{d} \widehat{x}_{i}<1\right\}$. By $h_{T}=\operatorname{diam}(T)$ we signify the element diameter of $T \in \mathcal{T}^{h}$, and by $h=\max _{T \in \mathcal{T}^{h}} h_{T}$ the mesh size. Furthermore, by $\mathcal{E}^{h}$ we denote the set of all interior mesh nodes for $d=1$ and interior (open) edges for $d=2$ in $\mathcal{T}^{h}$. In addition, for $T \in \mathcal{T}^{h}$, we let $\mathcal{E}^{h}(T)=\left\{E \in \mathcal{E}^{h}: E \subset \partial T\right\}$. For $E \in \mathcal{E}^{h}$, we let $h_{E}$ be the mean of the lengths of the adjacent elements in $1 \mathrm{~d}$, and the length of $E$ in $2 \mathrm{~d}$. Let us also define the following two quantities:

$$
\alpha_{T}:=\min \left(1, \varepsilon^{-1 / 2} h_{T}\right), \quad \alpha_{E}:=\min \left(1, \varepsilon^{-1 / 2} h_{E}\right),
$$

for $T \in \mathcal{T}^{h}$ and $E \in \mathcal{E}^{h}$, respectively.

We consider the finite element space of continuous, piecewise linear functions on $\mathcal{T}^{h}$ with zero trace on $\partial \Omega$, given by

$$
V_{0}^{h}:=\left\{\varphi \in H_{0}^{1}(\Omega):\left.\varphi\right|_{T} \in \mathbb{P}_{1}(T) \forall T \in \mathcal{T}^{h}\right\},
$$

respectively, where $\mathbb{P}_{1}(T)$ is the standard space of all linear polynomial functions on $T$.

3.1.2. Linear Finite Element Discretization. For $t=\frac{c}{L^{2}}$ and $u_{h}^{n} \in V_{0}^{h}, n \geq 0$, we consider the finite element approximation of (20), which is to find $u_{h}^{n+1} \in V_{0}^{h}$ such that

$$
\left(u_{h}^{n+1}, v_{h}\right)_{X}=\mathrm{B}_{\varepsilon}\left(u_{h}^{n}\right)\left(v_{h}\right), \quad \forall v_{h} \in V_{0}^{h},
$$

where, for a fixed $u_{h} \in V_{0}^{h}$,

$$
\mathrm{B}_{\varepsilon}\left(u_{h}\right)\left(v_{h}\right)=\left(u_{h}, v_{h}\right)_{X}-t \int_{\Omega}\left\{\varepsilon \nabla u_{h} \cdot \nabla v_{h}-f_{h}\left(u_{h}\right) v_{h}\right\} \mathrm{d} \boldsymbol{x} .
$$

Here, $f_{h}\left(u_{h}\right) \in V_{h}$ is defined through

$$
\int_{\Omega}\left(f\left(u_{h}\right)-f_{h}\left(u_{h}\right)\right) v_{h} \mathrm{~d} \boldsymbol{x}=0, \quad \forall v_{h} \in V_{0}^{h} .
$$

More precisely, if $V_{0}^{h}$ is spanned by the basis functions $\left\{\phi_{i}\right\}_{i=1}^{N}$ we solve the algebraic system

$$
\sum_{k=1}^{N} B_{k i} u_{k}^{n+1}=\sum_{k=1}^{N} B_{k i} u_{k}^{n}-t \varepsilon \sum_{k=1}^{N} A_{k i} u_{k}^{n}+t b\left(u_{h}^{n}\right)_{i}, \quad i \in\{1, \ldots, N\},
$$


with respect to $\left\{u_{k}^{n+1}\right\}_{k=1}^{N}$ and set $u_{h}^{n+1}=\sum_{k=1}^{N} u_{k}^{n+1} \phi_{k}$. In (25), $B$ signifies the iteration matrix given by $B_{i k}=\left(\phi_{i}, \phi_{k}\right)_{X}$ and $A$ denotes the stiffness matrix. Moreover, for $i=\{1, \ldots, N\}$ the load vector is given by $b\left(u_{h}^{n}\right)_{i}:=\int_{\Omega} f\left(u_{h}^{n}\right) \phi_{i} \mathrm{~d} \boldsymbol{x}$.

3.2. A Posteriori Analysis. The aim of this section is to derive a posteriori error bounds for the FEM iteration (24). In view of Proposition 2.5 it is sufficient to derive a computable quantity $\eta\left(u_{h}^{n+1}, V_{0}^{h}\right)$.

Therefore we introduce the quantity:

$$
\tilde{e}_{h}^{n+1, n}:=\tilde{u}^{n+1, n}-u_{h}^{n+1} .
$$

Moreover, let $\mathcal{I}^{h}: H_{0}^{1}(\Omega) \rightarrow V_{0}^{h}$ be the quasi-interpolation operator of Clément (see, e.g., [2, Corollary 4.2]) and set $v_{h}:=\mathcal{I}^{h} \tilde{e}_{h}^{n+1, n}$.

We observe

$$
\begin{aligned}
\left\|\tilde{e}_{h}^{n+1, n}\right\|_{\varepsilon}^{2}= & \left(\tilde{u}^{n+1, n}, \tilde{e}_{h}^{n+1, n}\right)_{X}-\left(u_{h}^{n+1}, \tilde{e}_{h}^{n+1, n}\right)_{X} \\
= & \left(\tilde{u}^{n+1, n}, \tilde{e}_{h}^{n+1, n}-v_{h}\right)_{X}-\left(u_{h}^{n+1}, \tilde{e}_{h}^{n+1, n}-v_{h}\right)_{X} \\
= & -t \int_{\Omega}\left\{\varepsilon \nabla u_{h}^{n} \nabla\left(\tilde{e}_{h}^{n+1, n}-v_{h}\right)-f\left(u_{h}^{n}\right)\left(\tilde{e}_{h}^{n+1, n}-v_{h}\right)\right\} \mathrm{d} \boldsymbol{x}-\left(u_{h}^{n+1}-u_{h}^{n}, \tilde{e}_{h}^{n+1, n}-v_{h}\right)_{X} \\
= & -\sum_{T \in \mathcal{T}_{h}} \int_{T}\left\{\varepsilon \nabla\left(u_{h}^{n+1}-u_{h}^{n}\right) \nabla\left(\tilde{e}_{h}^{n+1}-v_{h}\right)+t \varepsilon \nabla u_{h}^{n} \nabla\left(\tilde{e}_{h}^{n+1, n}-v_{h}\right)\right\} \mathrm{d} \boldsymbol{x} \\
& +\sum_{T \in \mathcal{T}_{h}} \int_{T}\left\{t f_{h}\left(u_{h}^{n}\right)\left(\tilde{e}_{h}^{n+1, n}-v_{h}\right)-\left(u_{h}^{n+1}-u_{h}^{n}\right)\left(\tilde{e}_{h}^{n+1, n}-v_{h}\right)\right\} \mathrm{d} \boldsymbol{x} \\
& +\sum_{T \in \mathcal{T}_{h}} \int_{T} t\left(f\left(u_{h}^{n}\right)-f_{h}\left(u_{h}^{n}\right)\right)\left(\tilde{e}_{h}^{n+1, n}-v_{h}\right) \mathrm{d} \boldsymbol{x} .
\end{aligned}
$$

Integrating by parts in the first term on the right-hand side, recalling the fact that $\left(v-\mathcal{I}^{h} v\right)=0$ on $\partial \Omega$, and applying some elementary calculations, yields that

where

$$
\left\|\tilde{e}_{h}^{n+1, n}\right\|_{\varepsilon}^{2}=\sum_{T \in \mathcal{T}_{h}}\left(b_{T}+c_{T}\right)+\sum_{E \in \mathcal{E}_{h}} a_{E}
$$

$$
\begin{aligned}
b_{T} & :=\int_{T}\left\{\left(\varepsilon \Delta\left(u_{h}^{n+1}-u_{h}^{n}\right)-\left(u_{h}^{n+1}-u_{h}^{n}\right)+t \varepsilon \Delta u_{h}^{n}+t f_{h}\left(u_{h}^{n}\right)\right)\left(\tilde{e}_{h}^{n+1, n}-v_{h}\right)\right\} \mathrm{d} \boldsymbol{x}, \\
a_{E} & :=\int_{\partial E} \varepsilon \llbracket \nabla\left(u_{h}^{n+1}-u_{h}^{n}\right)+t \nabla u_{h}^{n} \rrbracket\left(\tilde{e}_{h}^{n+1, n}-v_{h}\right) \mathrm{d} s, \\
c_{T} & :=\int_{T} t\left(f\left(u_{h}^{n}\right)-f_{h}\left(u_{h}^{n}\right)\right)\left(\tilde{e}_{h}^{n+1, n}-v_{h}\right) \mathrm{d} \boldsymbol{x} .
\end{aligned}
$$

with $E \in \mathcal{E}^{h}, T \in \mathcal{T}^{h}$. Here, for any edge $E=\partial T^{\sharp} \cap \partial T^{b} \in \mathcal{E}^{h}$ shared by two neighboring elements $T^{\sharp}, T^{b} \in \mathcal{T}^{h}$, where $\boldsymbol{n}^{\sharp}$ and $\boldsymbol{n}^{\mathrm{b}}$ signify the unit outward vectors on $\partial T^{\sharp}$ and $\partial T^{b}$, respectively, we denote by

$$
\llbracket \nabla u_{h}^{n+1} \rrbracket(\boldsymbol{x})=\lim _{t \rightarrow 0^{+}} \nabla u_{h}^{n+1}\left(\boldsymbol{x}+t \boldsymbol{n}^{\sharp}\right) \cdot \boldsymbol{n}^{\sharp}+\lim _{t \rightarrow 0^{+}} \nabla u_{h}^{n+1}\left(\boldsymbol{x}+t \boldsymbol{n}^{b}\right) \cdot \boldsymbol{n}^{b}, \quad \boldsymbol{x} \in E,
$$

the jump across $E$.

Then, for $T \in \mathcal{T}^{h}$, defining the FEM-error

$$
\begin{aligned}
\eta_{n+1, T}^{2}:= & \alpha_{T}^{2}\left\|\varepsilon \Delta\left(u_{h}^{n+1}-u_{h}^{n}\right)-\left(u_{h}^{n+1}-u_{h}^{n}\right)+t \varepsilon \Delta u_{h}^{n}+t f_{h}\left(u_{h}^{n}\right)\right\|_{0, T}^{2} \\
& +\frac{1}{2} \sum_{E \in \mathcal{E}^{h}(T)} \varepsilon^{-1 / 2} \alpha_{E}\left\|\varepsilon \llbracket \nabla\left(u_{h}^{n+1}-u_{h}^{n}\right)+t \nabla u_{h}^{n} \rrbracket\right\|_{0, E}^{2} .
\end{aligned}
$$

with $\alpha_{T}$ and $\alpha_{E}$ from (23), we proceed along the lines of the proof of [2, Theorem 4.4] in order to obtain the following result. 
Proposition 3.2. For $n \geq 0$ there holds the upper a posteriori bound

$$
\left\|\tilde{e}_{h}^{n+1, n}\right\|_{\varepsilon} \preccurlyeq\left(t\left\|f\left(u_{h}^{n}\right)-f_{h}\left(u_{h}^{n}\right)\right\|_{0, \Omega}^{2}+\sum_{T \in \mathcal{T}_{h}} \eta_{n+1, T}^{2}\right)^{1 / 2}=: \eta\left(u_{h}^{n+1}, V_{0}^{h}\right)
$$

with $\eta_{n+1, T}, T \in \mathcal{T}^{h}$ from $(26)$.

Using Proposition 2.5 together with the bound (27), we end up with the following a posteriori error bound:

Theorem 3.3. For $n \geq 0$ there holds the upper a posteriori error bound

$$
\left\|e_{h}^{n+1}\right\|_{\varepsilon} \preccurlyeq \eta\left(u_{h}^{n+1}, V_{0}^{h}\right)+\eta_{\mathrm{FP}}\left(u_{h}^{n+1}, u_{h}^{n}, V_{0}^{h}\right),
$$

with

$$
\eta_{\mathrm{FP}}\left(u_{h}^{n+1}, u_{h}^{n}, V_{0}^{h}\right):=\left\|u_{h}^{n+1}-u_{h}^{n}\right\|_{\varepsilon},
$$

$T \in \mathcal{T}^{h}$ and $\eta\left(u_{h}^{n+1}, V_{0}^{h}\right)$, from $(27)$, respectively.

3.3. A Fully Adaptive Fixed-Point-Galerkin Algorithm. We will now propose a procedure that will combine the fixed point iteration procedure with an automatic finite element mesh refinement strategy. More precisely, based on the a posteriori error bound from Theorem 3.3, the main idea of our approach is to provide an interplay between the fixed point iterations and adaptive mesh refinements which is based on monitoring the error indicators in (29) and (27), and on acting according to whatever quantity is dominant in the current computations.

The individual computational steps are summarized in Algorithm 1.

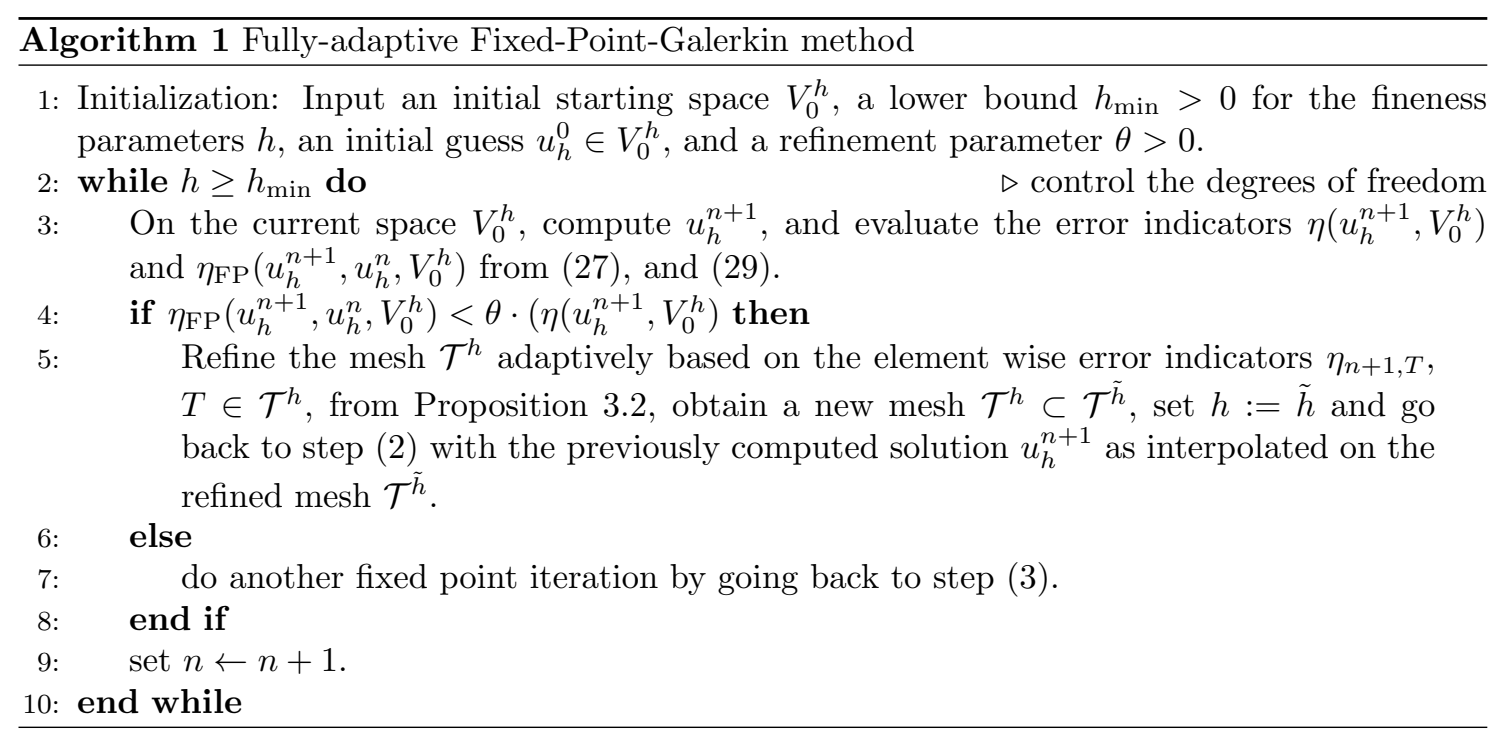

\section{Numerical ExPERIMENT}

We will now illustrate and test the above fully adaptive Algorithm 1 with a numerical experiment in 2 d. The linear systems resulting from the finite element discretization (25) are solved by means of a direct solver.

Example 4.1. We consider the problem

$$
-\varepsilon \Delta u=f(u), \quad \text { in } \Omega=(0,1)^{2}, \quad u=0 \quad \text { on } \partial \Omega,
$$

with $f(u)=\frac{1-u}{1+\mathrm{e}^{-(u-1)^{2}}}$. Here $\left|\partial_{u} f(u)\right|$ is uniformely bounded (roughly by 1.3 ) and

$$
\partial_{u} f(u) \leq\left.\partial_{u} f(u)\right|_{u=1}=-1 / 2 .
$$

Henceforth we have $L=L_{f} \approx 1.3, c=c_{f}=1 / 2$, i.e., the problem is well defined. 

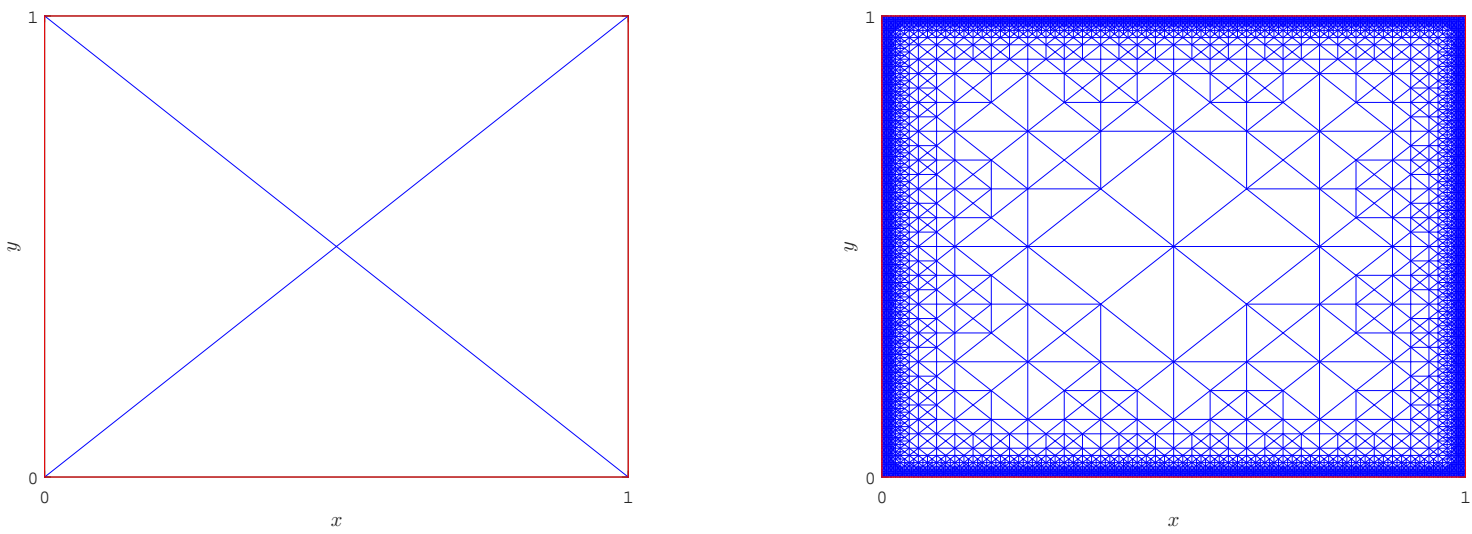

Figure 3. Example 4.1 for $\varepsilon=10^{-7}$ : Initial mesh for the numerical solution (left), and the mesh corresponding to the numerical solution (right).

The focus of this experiment is on the robustness of the a posteriori error bound (3.3) with respect to the singular perturbation parameter $\varepsilon$ as $\varepsilon \rightarrow 0$. Indeed, neglecting the boundary conditions for a moment, one observes that the unique positive zero $u=1$ of $f(u)$ is a solution of the PDE. We therefore expect boundary layers along $\partial \Omega$; see Figures 3 and 4 (right).

Starting from the initial mesh depicted in Figure 3 (left) with $u_{0}^{h}(1 / 2,1 / 2)=1$, we test the fully adaptive fixed point Galerkin Algorithm 1 for different choices of $\varepsilon=\left\{10^{-i}\right\}_{i=0}^{8}$. In Algorithm 1 the parameter $\theta$ is chosen to be 0.5. Furthermore, in this example, the procedure is always initiated with a uniform initial mesh $\mathcal{T}$ consisting of 4 elements; see Figure 3 (left). As $\varepsilon \rightarrow 0$ the resulting solutions feature ever stronger boundary layers; see Figures 3 and 4 (right). Again we see that the performance data in Figure 4 shows errors decay, firstly, robust in $\varepsilon$, and, secondly, of (optimal) order $1 / 2$ with respect to the number of degrees of freedom.
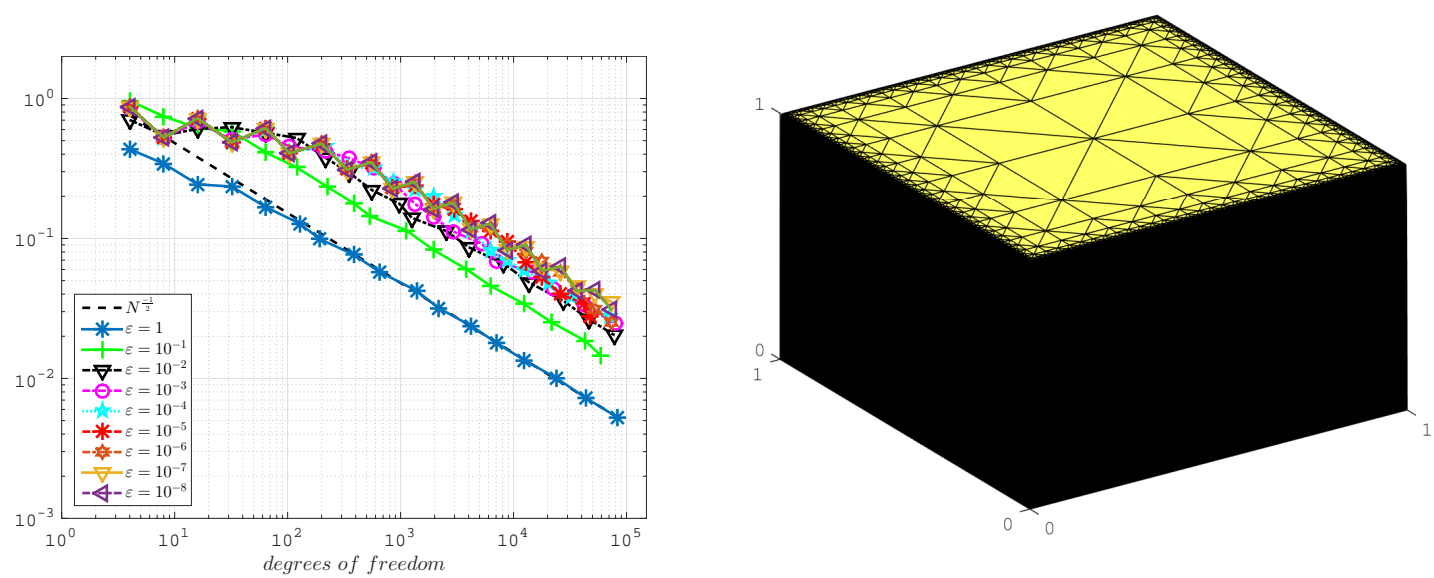

Figure 4. Example 4.1: Estimated errors for different choices of $\varepsilon$ (left) and the numerical solution of 30 for $\varepsilon \ll 1$ (right).

\section{Conclusions}

The aim of this paper is to introduce a reliable and computationally feasible procedure for the numerical solution of semilinear elliptic boundary value problems, with possible singular perturbations. The key idea is to combine a simple fixed point method with an automatic mesh refinement finite element procedure. Furthermore, the sequence of linear problems resulting from 
the application of the fixed point iteration and Galerkin discretization is treated by means of a robust (with respect to the singular perturbations) a posteriori error analysis and a corresponding adaptive mesh refinement process. Our numerical experiments clearly illustrate the ability of our approach to reliably find the solution of the underlying well posed problem, and to robustly resolve the singular perturbations at an optimal rate.

\section{REFERENCES}

1. M. Amrein, J. M. Melenk, and T. P. Wihler, An hp-adaptive Newton-Galerkin finite element procedure for semilinear boundary value problems, Mathematical Methods in the Applied Sciences 40 (2016), no. 6, 19731985, 13 pages, mma.4113.

2. M. Amrein and T. P. Wihler, Fully adaptive Newton-Galerkin methods for semilinear elliptic partial differential equations, SIAM J. Sci. Comput. 37 (2015), no. 4, A1637-A1657.

3. M. Amrein and T. P. Wihler, Fully adaptive Newton-Galerkin time stepping methods for singularly perturbed parabolic evolution equations, IMA, Journal of Numerical Analysis, 15 pages (in press), 2016.

4. __ Fully adaptive pseudo transient continuation Galerkin methods for semilinear elliptic partial differential equations, Accepted for publication in Numerical Methods for Partial Differential Equations, 15 pages (in press), 2017.

5. G. Barles and J. Burdeau, The Dirichlet problem for semilinear second-order degenerate elliptic equations and applications to stochastic exit time control problems, Comm. Partial Differential Equations 20 (1995), no. 1-2, $129-178$.

6. H. Berestycki and P.-L. Lions, Nonlinear scalar field equations. I. Existence of a ground state, Arch. Rational Mech. Anal. 82 (1983), no. 4, 313-345.

7. R. S. Cantrell and C. Cosner, Spatial ecology via reaction-diffusion equations, Wiley Series in Mathematical and Computational Biology, John Wiley \& Sons, Ltd., Chichester, 2003.

8. A. Chaillou and M. Suri, A posteriori estimation of the linearization error for strongly monotone nonlinear operators, Journal of Computational and Applied Mathematics 205 (2007), no. 1, 72-87.

9. S. Congreve and T. P. Wihler, An iterative finite element method for strongly monotone quasi-linear diffusionreaction problems, Tech. Report 1506.08851, arXiv.org, 2015.

10. P. Deuflhard, Newtons method for nonlinear problems, Springer Ser. Comput. Math., 2004.

11. L. Edelstein-Keshet, Mathematical models in biology, Classics in Applied Mathematics, vol. 46, Society for Industrial and Applied Mathematics (SIAM), Philadelphia, PA, 2005, Reprint of the 1988 original.

12. L. El Alaoui, A. Ern, and M. Vohralík, Guaranteed and robust a posteriori error estimates and balancing discretization and linearization errors for monotone nonlinear problems, Computer Methods in Applied Mechanics and Engineering 200 (2011), no. 37-40, 2782-2795.

13. A. Ern and M. Vohralík, Adaptive inexact Newton methods with a posteriori stopping criteria for nonlinear diffusion PDEs, SIAM J. Sci. Comput. 35 (2013), no. 4, A1761-A1791.

14. Lawrence C. Evans, Partial differential equations, Graduate studies in mathematics, American Mathematical Society, Providence (R.I.), 1998, RÃ Cimpr. avec corrections : 1999, 2002.

15. A. Friedman (ed.), Tutorials in mathematical biosciences. IV, Lecture Notes in Mathematics, vol. 1922, Springer, Berlin; MBI Mathematical Biosciences Institute, Ohio State University, Columbus, OH, 2008, Evolution and ecology, Mathematical Biosciences Subseries.

16. E. M. Garau, P. Morin, and C. Zuppa, Convergence of an adaptive Kačanov FEM for quasi-linear problems, Applied Numerical Mathematics. 61 (2011), no. 4, 512-529.

17. P. Houston and T. P. Wihler, An hp -adaptive Newton-Discontinuous-Galerkin finite element approach for semilinear elliptic boundary value problems, Tech. report, http://arxiv.org, 2016.

18. W.-M. Ni, The mathematics of diffusion, CBMS-NSF Regional Conference Series in Applied Mathematics, vol. 82, Society for Industrial and Applied Mathematics (SIAM), Philadelphia, PA, 2011.

19. A. Okubo and S. A. Levin, Diffusion and ecological problems: modern perspectives, second ed., Interdisciplinary Applied Mathematics, vol. 14, Springer-Verlag, New York, 2001.

20. W. A. Strauss, Existence of solitary waves in higher dimensions, Comm. Math. Phys. 55 (1977), no. 2, $149-162$.

21. Eberhard Zeidler, Nonlinear Functional Analysis and its Applications. I, Springer-Verlag, New York, 1986, Fixed-point theorems. 